\title{
LHomme
}

L'HOMME Revue française d'anthropologie

$190 \mid 2009$

Varia

\section{Ruth Finnegan, The Hidden Musicians}

\section{Charlotte Poulet}

\section{(2) OpenEdition}

Journals

Édition électronique

URL : http://journals.openedition.org/lhomme/28747

DOI : $10.4000 /$ /homme.28747

ISSN : 1953-8103

\section{Éditeur}

Éditions de l'EHESS

\section{Édition imprimée}

Date de publication : 1 janvier 2009

Pagination : 241-245

ISSN : 0439-4216

\section{Référence électronique}

Charlotte Poulet, «Ruth Finnegan, The Hidden Musicians », L'Homme [En ligne], 190 | 2009, mis en ligne le 03 janvier 2017, consulté le 23 septembre 2020. URL : http://journals.openedition.org/lhomme/ 28747 ; DOI : https://doi.org/10.4000//homme.28747

Ce document a été généré automatiquement le 23 septembre 2020.

(c) École des hautes études en sciences sociales 


\title{
Ruth Finnegan, The Hidden Musicians
}

\author{
Charlotte Poulet
}

\section{RÉFÉRENCE}

Ruth Finnegan, The Hidden Musicians. Music-Making in an English Town, Middletown, Wesleyan University Press, 2007, XXIII + 378 p.

1 RUTH FINNEGAN nous propose dans cet ouvrage une anthropologie du proche. C'est dans la ville même où elle réside, Milton Keynes, qu'elle poursuit l'analyse de ses thèmes de prédilections: l'oralité et la performance. Au début des années 1980, l'auteure y découvre l'intensité de la vie musicale et ses multiples manifestations: concerts, messes musicales, fanfares, etc. Elle souhaite dès lors en saisir les tenants et aboutissants. Finalement, nous pourrions résumer ainsi la question posée ici : pourquoi fait-on de la musique? Pour y répondre, l'auteure s'intéresse aux acteurs de cette pratique, les musiciens "cachés ». Ce terme désigne les musiciens amateurs et a pour vocation de dénoncer le trop peu d'intérêt porté à la production locale de la musique. L'originalité de cette démarche tient au fait de penser cette production comme facette invisible, mais active, de la culture urbaine et nationale anglaise, de penser tous ces petits « gestes » musicaux comme participant, tant aux changements, qu'à la continuité de la musique anglaise d'aujourd'hui. Toutefois, le terme « amateur » est bien difficile à définir; Ruth Finnegan en choisit elle-même le contenu en se focalisant sur les musiciens ordinaires, ceux qui animent la production locale, au-delà de la distinction par la rémunération. Cet ouvrage n'est pas l'étude d'une pratique musicale particulière, ni celle de la musique en tant que produit, mais bien l'ethnographie de pratiques collectives locales dans leur diversité, en un lieu et un temps donnés. Le point de vue anthropologique de l'auteure tend à considérer ces pratiques dans un ensemble plus vaste, un «monde musical» (pp.31-32) répondant à des règles sociales et à une organisation collective.

2 C'est ce « monde » que Ruth Finnegan nous dépeint. Pour ce faire, elle sélectionne des activités locales: la musique classique, les fanfares, la musique folk, les comédies 
musicales, le jazz, la musique country et le rock, qui tour à tour seront décrites précisément à travers leurs acteurs, leurs organisations, leurs lieux, leurs enseignements et leurs règles. Grâce à cette ethnographie systématique l'auteure établit des critères, communs à toutes pratiques, lui permettant de les comparer. Tout d'abord, elle les distingue par leur mode d'apprentissage, formel ou informel, tout en expliquant que ce sont les conventions sociales établies autour de chaque pratique qui en déterminent la forme et non les spécificités des musiques évoquées. Puis, elle les différencie par les différents types de performance mis en place par les acteurs, en prenant donc en compte les musiciens tout autant que le public. Chaque musique se produit différemment, s'écoute et se fait d'une manière distincte. Enfin, ces pratiques musicales sont comparées par la manière dont la création et l'innovation s'établissent au sein de leur tradition. La conclusion de ce travail amène à repenser le "monde » musical de Milton Keynes comme des «mondes pluriels» (p. 180) s'unissant et se distinguant autour de leurs conventions implicites pour la transmission, la performance et la création.

3 Face à la pluralité de ces «mondes", Ruth Finnegan cherche à comprendre l'implication des institutions et le rôle de l'organisation de ces événements dans le maintien de cette effervescence musicale. Les institutions en question sont bien plus que des structures puisqu'elles sont des lieux où la musique bat son plein, tels que le pub, la maison familiale, l'église et l'école. Elles permettent le financement et l'organisation de bien des pratiques musicales ainsi qu'une socialisation musicale de l'individu dès son plus jeune âge. Ces lieux sont ceux de la vie locale, et la musique y tient une place importante. Enfin, pour clore ce panorama, soulignons que la production musicale dépend d'un réseau complexe d'institutions, ce n'est pas parce qu'elle est non professionnelle et locale qu'elle en est moins organisée. Elle nécessite une structure et un investissement actif de la part de ses membres. La musique locale n'est pas spontanée, donnée et gratuite. Elle ne survit que par les efforts individuels mis en place.

4 Pour conclure son étude, Ruth Finnegan s'interroge sur l'impact des pratiques musicales sur la vie locale en général en revenant sur ses découvertes. Elle note que, contrairement à son hypothèse de départ, il n'y a pas de réelle dimension communautaire, ni de sens de l'appartenance locale, dans la pratique musicale. L'activité musicale ressemble, bien plus, selon elle, à la vie urbaine: moderne, impersonnelle et anonyme. La pratique ne donne pas lieu à un monde social partagé localement. Les «mondes» décrits précédemment sont plutôt de l'ordre de l'hétérogénéité, de la flexibilité, de l'ouverture. Toutefois, la pratique musicale demeure une action collective par laquelle on partage. C'est donc la notion même de «monde » qui est remise en cause par le manque de place laissée à l'individu et à ses choix, car c'est bien lui qui, par son investissement, contribue à la continuité de la vie musicale locale. Ruth Finnegan parlera désormais de "sentier» musical (pathways, p. 297) parcourant la vie urbaine moderne et sur lequel chacun peut voguer au gré de la musique. Ce "sentier» musical n'est donc pas communautaire mais engendre des espaces et des temps collectifs. Il résulte tant de facteurs sociaux (socialisation, genre et âge) que de choix individuels. Cette conclusion a pour intérêt de redéfinir le « local ", qui n'est plus ici simplement topographique mais s'inscrit dans l'espace par un ensemble de pratiques et de relations sociales. La pratique musicale devient un lieu, 
crée un espace, source de socialités. C'est ainsi que la pratique de la musique rythme la vie locale et crée du lien social.

5 La réédition de cet ouvrage ne nous donne pas à lire une étude dépassée. En effet, même si la musique suit son cours, elle continue d'être pratiquée jour après jour. C'est ainsi qu'en nous présentant une ethnographie de la pratique musicale en général en un lieu donné, Ruth Finnegan apporte des éléments théoriques à l'anthropologie de la musique. Elle nous démontre habilement que les activités musicales ne sont pas des événements individuels et isolés mais relèvent d'une participation à la vie locale dans son ensemble. Elles forment un tout au-delà de ce qui les différencie. L'auteure prouve ici l'intérêt d'une anthropologie de la pratique. Le son produit ne nous en dirait pas plus, c'est la production même de ce son et son contexte qui est le mieux à même de nous éclairer sur les processus sociaux mis en œuvre. Cet ouvrage pose également un jalon, qui ne pourra perdre en pertinence, en traitant la musique amateur locale comme le laboratoire de la culture anglaise, le lieu de l'expression musicale.

On regrettera toutefois l'absence du discours des principaux intéressés malgré les nombreux entretiens que l'auteure a pu réaliser. En effet, la subjectivité de ces mots aurait pu apporter un autre éclairage sur de nombreux points. On regrettera également le peu de place accordée aux " amateurs écoutants ", car eux aussi, à leur manière, sont pleinement participants, et font vivre la vie musicale locale. Sans public, il n'y a pas d'effervescence musicale. Cet élargissement de la notion aurait peut-être fait apparaître un autre genre de pratique, la pratique spontanée (Ruth Finnegan cite ellemême brièvement les singsongs), celle qui ne nécessite pas forcément d'organisation, mais qui participe tout autant à la vie locale qu'à la socialisation musicale.

\section{AUTEURS}

\section{CHARLOTTE POULET}

Laboratoire d'anthropologie et d'histoire de l'institution de la culture, Paris. charlottepoulet@free.fr 